\title{
APRENDIENDO LA "R": RACIALIZACIÓN Y RACISMO PROSAICO EN ESCUELAS BOGOTANAS
}

\section{Learning the "R": Racialisation and Prosaic Racism within Schools in Bogota}

\section{OSCAR QUINTERO RAMÍREZ*}

Fecha de recepción: 04 de agosto de 2017 - Fecha de aprobación: 18 de octubre de 2017

\section{Resumen}

El artículo presenta los resultados de una investigación cualitativa en donde se retoman los relatos biográficos de jóvenes afrocolombianos y afrocolombianas sobre sus trayectorias educativas. El análisis se centra en sus experiencias vividas de racismo, en el contexto escolar, en la ciudad de Bogotá. Se identificaron dinámicas que vivieron estas personas desde su primera infancia, enfrentándolas a lo que proponemos comprender en términos de racismo prosaico: entendido como esa temprana racialización, vivida especialmente en la infancia, cargada de poderosa violencia simbólica y, muchas veces, física, que da paso a la construcción de una alteridad radical que es corporal, racializada y jerarquizada. Su expresión es, por lo general, explícita, vulgar y violenta; así como es traumática y dolorosa para los sujetos racializados como "negros" y "negras".

Palabras clave: racismo, escuelas, Bogotá, infancia, racismo prosaico

\section{Abstract}

This article presents the results of a qualitative research based on several interviews to male and female young afrocolombians who talked about their educational trajectories. The analysis is focused on their lived experiences about racism within the scholar context in Bogota city. We have identified some dynamics that they had to face from their very childhood, experiencing what we aim to call as prosaic racism: meaning as that first racialization, lived basically when they are children, charged with a powerful symbolic, and also physical, violence that leads to a construction of a radical alterity which is bodily, racialized and hierarchical. Generally, it is explicit, vulgar and violent expressed; as well as traumatic and painful for the people racialized in terms of "black".

Keywords: racism, schools, Bogota, Childhood, prosaic racism

* Dr. en Sociología. Profesor Asistente, Departamento de Sociología, Investigador del Centro de Estudios Sociales, Universidad Nacional de Colombia, Bogotá, Colombia. Artículo enmarcado en tesis doctoral, financiada por el IRD-Francia. Correo-e: oaquinteror@unal.edu.co,oquinteror@yahoo.com 
Entre etnicidad y racialización: el estudio de las poblaciones "negras" o "afrocolombianas"

En Colombia, a pesar del reconocimiento de la ciudadanía a partir de la diferencia cultural y de los derechos sociales, económicos y políticos, las desigualdades sociales son cada vez más profundas en el conjunto de la sociedad, afectando de maneras más intensas a los grupos étnicos. Así lo corrobora un estudio de la Comisión Interamericana de Derechos Humanos (Universidad de Texas, 2007), en donde se identifican varias trabas para el pleno ejercicio de los derechos a causa de los actores armados, la industria del turismo, la agroindustria, el comercio ilegal de drogas, así como las industrias extractivistas, en general. A esto se suma la poca claridad, voluntad política y decisión del Estado para prevenir, investigar y sancionar las violaciones a estos derechos y para hacer respetar la legislación nacional e internacional.

Uno de los resultados de esta situación ha sido la creciente presencia, en las principales ciudades del país, de personas en situación de desplazamiento a causa de la violencia, de los cuales una alta proporción son de las "comunidades negras" 1 y quienes se deben enfrentar a procesos de discriminación en las urbes (Rodríguez Garavito, Alfonso \& Cavelier, 2008). Para el caso de las poblaciones "afrocolombianas", una de las consecuencias de esta situación desigual ha sido la transformación de las luchas por la tierra en desafíos identitarios que ponen en cuestión la definición misma de la supuesta identidad nacional (Agier \& Hoffmann 1999; Hoffmann, 2004).

La identidad se ha convertido entonces en una categoría central en el estudio de las minorías étnicas y de las poblaciones "negras" en Colom- bia, principalmente desde los años 1990. No obstante, el camino recorrido para llegar hasta allí ha estado marcado por diversas corrientes de estudio, algunas de ellas contradictorias entre sí. En ese sentido, cabe preguntarse, ¿cómo se convirtió "lo negro", las poblaciones "negras" o "afrocolombianas" en una preocupación de estudio para las ciencias sociales en Colombia?, ¿Cuáles han sido las orientaciones conceptuales (y disciplinarias si se quiere) que han guiado estos estudios?

En primer lugar, es importante anotar que existe un corpus consolidado de investigaciones sobre las poblaciones "negras" en las ciencias sociales colombianas, así como también puede afirmarse que existe una "comunidad académica"; lo cual se conoce más comúnmente bajo la etiqueta de "estudios afrocolombianos"2. Los primeros estudios sobre estas poblaciones fueron desarrollados principalmente desde la antropología, presentando un fuerte "déficit" y "retraso" en relación con la investigación antropológica desarrollada hasta entonces sobre los indígenas. Al decir de Restrepo (1999), "esta asimetría en la producción antropológica es la expresión de una disciplina antropológica que hallaba en lo indio su paradigma de análisis" (p. 2) Una asimetría que había sido construida histórica y socialmente en el conjunto de la sociedad colombiana y que se había mantenido en el campo académico.

Sin querer simplificar demasiado, podría decirse que han existido dos tendencias generales en los estudios sobre las poblaciones "negras" en Colombia. Por un lado, algunos autores han enfatizado en análisis de tipo cultural e histórico, mientras que, otros, han desarrollado preguntas de investigación que desde la perspectiva histórica-cultural no podían ser 
tratadas. Es lo que podría denominarse como una perspectiva "etnicista" y otra perspectiva más centrada sobre los procesos de "racialización", de los cuales son objeto estas poblaciones. No sobra mencionar que estas dos perspectivas no son necesariamente contradictorias entre sí; el mayor o menor grado de cada una en los estudios específicos depende de los intereses particulares de investigadores o grupos de investigación, de los marcos conceptuales empleados, así como de las tendencias disciplinarias que los animan.

En ese sentido, uno de los aspectos que es necesario resaltar tiene que ver con la falta de unanimidad en la definición del objeto de estudio dentro de las diferentes tendencias de investigación. Según Restrepo (2004), la pluralidad en la definición del objeto de estudio se encuentra principalmente en los autores que han trabajado desde la perspectiva histórico-cultural. No obstante, al revisar los trabajos pertenecientes a la otra tendencia de estudios, la "etnicista", se puede apreciar que también hay una diversidad en la definición del objeto de estudio, aunque esta sí es más limitada. Esta pluralidad de definiciones se ve reflejada en los distintos términos elaborados, empleados y re-elaborados en los distintos estudios, como lo son "poblaciones, grupos, sociedades, comunidades, cultura/s, raza o etnia/s puntuadas de negras, afrocolombianas, afrodescendientes o negroides" (Restrepo, 2004, p. 6). A su vez, estas definiciones están relacionadas con las principales orientaciones conceptuales que han guiado las investigaciones.

En una de la compilaciones bibliográficas más recientes y completas que se puedan tener (a pesar de los límites que una tal compilación pueda presentar) sobre la producción acadé- mica en este campo de estudios (Restrepo \& Rojas, 2008), tan solo se encuentran 15 títulos con la palabra "discriminación" y 12 títulos con la palabra "racismo", dentro de un total aproximado de 1.870 referencias citadas en la compilación, las cuales incluyen libros completos, artículos de revista, artículos en libro colectivo, tesis de grado y "documentación gris", que son documentos no publicados de instituciones $u$ organizaciones (Restrepo \& Rojas, 2008).

A pesar de que el racismo y las discriminaciones son temas menores en el campo de los "estudios afrocolombianos", existen trabajos que permiten identificar un sub-campo en ciernes, con participación de investigadores e investigadoras que han acumulado producción y años de investigación (Mosquera, 1998; Urrea, 2011; Viveros \& Urrea, 2007; Viáfara \& Urrea, 2006; Meertens, Viveros \& Arango, 2006). A continuación, veremos con más detalle algunos de esos trabajos, en el sentido de una necesaria aproximación al estado del arte sobre el racismo y las discriminaciones en Colombia.

\section{Hacia un campo de estudios sobre el racismo y las discriminaciones en Colombia}

Una de las revistas de ciencias sociales más importantes en el campo académico de Colombia publicó en 2007 dos números centrados en la temática de "Raza y Nación", motivado por:

La creciente importancia de un aspecto cuya relevancia histórica, social y cultural es cada vez más evidente. En un país en donde la trascendencia del problema de la discriminación basada en criterios raciales no ha sido cabalmente reconocida, hasta el punto de llegar a pensarse que no existe, resulta refrescante y bienvenido el análisis riguroso del tema (Langebaek \& Leal, 2007, p. 11). 
El análisis de los artículos allí publicados muestra, sin embargo, una fuerte presencia de estudios de corte histórico, en donde se analiza el surgimiento de los discursos racialistas desde una perspectiva de la "formación del pensamiento racial en Colombia" y en donde se le presta una atención mayor a las "representaciones raciales", vistas desde el análisis de las percepciones, los estereotipos y los prejuicios. El análisis del racismo, desde una perspectiva más contemporánea, y si se quiere, mediante estudios empíricos, sigue siendo minoritario en este campo de estudios. En ese sentido, solo uno de los ocho trabajos publicados en la edición 27 de dicha revista formula, explícitamente, el tema de la discriminación racial en su título (Viveros, 2007) y de otros dos puede decirse que se aproximan a la problemática de forma implícita (Arocha, Guevara, Londoño, Moreno \& Rincón, 2007; Mosquera, 2007).

En el primero de ellos, se aborda directamente el tema de la discriminación racial en Bogotá, mediante un estudio de caso del ejercicio de la acción pública en una de las localidades más pobres de la ciudad, con gran participación demográfica de población "afrocolombiana". Se trata de estudiar las experiencias de los funcionarios, encargados de desarrollar las políticas públicas y sociales en esa entidad territorial, en relación con las poblaciones "afrocolombianas" beneficiarias de dichas políticas. La autora concluye que, a pesar de que en el país y en la ciudad se han producido avances normativos y promulgado leyes que prohíben cualquier tipo de discriminación, esta forma de relación social no ha desaparecido, así como tampoco ha sido objeto de muchos debates políticos ni académicos. En relación con los funcionarios objeto de estudio, se encontró una fuerte reticencia a hablar sobre temáticas relacionadas con la discriminación racial, así como a aceptar la existencia de prácticas institucionales de la misma, lo cual la autora analiza en términos de "denegación" del racismo y las discriminaciones (Viveros, 2007, p. 119).

Esta perspectiva de análisis es, en cierta forma, similar a la de Mosquera (2007) quien analiza los discursos y prácticas sociales de funcionarios públicos en programas de intervención social, especialmente en "Talleres de salud sexual y reproductiva y de fortalecimiento cultural", ofrecidos a "mujeres negras" que viven en Bogotá, como resultado de su desplazamiento forzado a causa de la violencia en los campos del país. La autora muestra cómo los discursos y las prácticas que componen esos talleres alimentan las asimetrías en las relaciones étnico raciales con la gente "negra", reproduciendo "prácticas racistas, sexistas y etnocéntricas que sitúan la identidad cultural, racial y personal de las mujeres "negras" desterradas, en un lugar inferior. Así mismo, según la autora, se refuerza la ideología del mestizaje al negar, no solo el "racismo social y cotidiano" que sufren algunas mujeres sino que se intenta moldear el comportamiento de la mujer "negra", considerado "agresivo, violento e indomable" (Mosquera, 2007, p. 134).

El artículo de Arocha et al. (2007) se propone analizar los procesos de invisibilidad y estereotipia como manifestaciones importantes del racismo en Colombia. En un proceso de formación de docentes de Bogotá, sobre la aplicación de la Cátedra de Estudios Afrocolombianos en las escuelas y colegios, los autores identifican el carácter paradójico de una tal acción pública. Si bien con esta Cátedra se propone promover la valoración y el respeto de los "afrodescendientes" y sus aportes a la nación, las acciones 
pedagógicas desarrolladas para este fin, efectivamente, mejoraban la visibilidad de África y de lo "afrocolombiano", pero a costa de reforzar y recrear estereotipos muy arraigados en torno a los africanos y "lo negro", como resultado de otra idea muy arraigada en la idiosincrasia colombiana, la de la supuesta superioridad de "lo blanco".

El aporte de este tipo de estudios al conocimiento sobre las dinámicas del racismo contemporáneo y los procesos de discriminación son innegables; se puede apreciar que, la mayoría de ellos, se enfoca en un estudio de las "representaciones raciales", vistas a través del "comportamiento" de los "racialmente dominantes" y de sus "prejuicios y estereotipos" inherentes. Una perspectiva tal es, desde luego, muy interesante y enriquecedora, pero no nos permite abordar la problemática del racismo y las discriminaciones desde la experiencia vivida por aquellas personas que son discriminadas, perspectiva que es central en el presente artículo.

En ese sentido, el campo de estudios sobre el racismo y las discriminaciones está en ciernes; hay argumentos que permiten augurar un mayor desarrollo desde múltiples perspectivas disciplinarias y enfoques de análisis (Fonseca \& Badrán, 2012). Nos referimos, principalmente, al creciente número de trabajos de pregrado y de maestría que se vienen desarrollando en varias universidades del país, así como al surgimiento de grupos de investigación preocupados por estas temáticas. Se destacan algunos estudios que abordan el racismo desde la lingüística y desde el análisis del discurso, así como desde la antropología. Estos trabajos se han centrado más en la experiencia vivida del racismo (Essed, 1991) que es la perspectiva que proponemos retomar en esta investigación. Por ese motivo, citamos algunos de esos trabajos.

El estudio de Fonseca (2009) busca analizar las percepciones del racismo que tienen los habitantes de Cartagena, por medio del "auto-análisis" que hacen de sus propias experiencias cotidianas de confrontación con situaciones que definen como racistas. Estos relatos se recaban mediante entrevistas en profundidad con "personas negras o mulatas, con cierto nivel previo de conciencia de la problemática racial". Nos parece que la definición a priori de esta "conciencia" es algo problemática, más bien debería ser un resultado de la investigación misma y no un "criterio" de selección de las personas participantes en la investigación. En ese sentido, ¿cómo se define esa conciencia, con qué parámetros? En el estudio en cuestión, las personas con esa conciencia son militantes en organizaciones con reivindicaciones étnicas. Se trataría entonces, en el mejor de los casos, de un tipo específico de conciencia, el de las personas "negras" o "mulatas" militantes, lo que nos llevaría a hablar en términos de conciencias ad infinitum, según las múltiples adscripciones socio-identitarias que puedan tener las personas o grupos sociales.

A pesar de este desacuerdo, que obedece más al nivel metodológico de la investigación, este trabajo es rico en aprendizajes relacionados con el fenómeno del racismo, en un contexto tan marcadamente inequitativo como el cartagenero. La autora concluye que el saber que los actores racializados tienen sobre el racismo es socialmente pertinente; en ese sentido, sus relatos sobre el racismo "son más que historias personales y remiten a experiencias sociales, que movilizan un sistema de conocimientos y de valores, sistemas normativos, estructuras de 
exclusión; el análisis sistemático de las percepciones individuales y las comparaciones intersubjetivas permiten acercarse a los escenarios del racismo, a las interpretaciones compartidas" (Fonseca, 2009, pp. 9-10). Por otro lado, en Cartagena las personas "negras" perciben la subsistencia del racismo, manifestado mediante prácticas verbales y formas de trato no igualitarias, expresadas al margen de las normas sociales y legales. La discriminación racial se sufre y se consolida como racismo cotidiano y frecuente; "las víctimas" así lo entienden y, en la mayoría de los casos, formulan juicios que sancionan moral y socialmente el comportamiento de quienes ejercen esas prácticas discriminatorias (Fonseca, 2009).

A partir de un análisis crítico del discurso, De la Hoz (2012) describe las opciones semánticas empleadas por "descendientes de africanos", al narrar experiencias de racismo en Cartagena, para dar a conocer los contenidos ideológicos subyacentes a dichas opciones. El estudio arrojó como resultado el uso de una amplia terminología colorista, que tiene la función -en la interacción social- de distinguir y diferenciar. Estas distinciones implican, sobre todo, una jerarquización y exclusión. Por otro lado, revelan el enorme peso de las percepciones de "los otros" en la construcción de la identidad del individuo; percepción hecha a partir de los rasgos fenotípicos (o somáticos para utilizar la expresión de Guillaumin (2008)). Esta situación es la que se da en la interacción social, a través de concepciones estéticas corporales, las cuales constituyen la naturalización de las diferencias socio-económicas, a través de la atribución estética de la fealdad y la belleza. En ese sentido, la atribución de la fealdad a ciertas condiciones sociales asociadas a rasgos corporales, contribuye a la cons- titución de una identidad deteriorada, es decir, estigmatizada (Goffman, 1970).

Desde una perspectiva más socio-antropológica, Gil (2010) aborda los temas de la discriminación racial y del racismo, concentrándose en las relaciones entre clase y "raza". Sus inquietudes giran en torno a la movilidad socio-económica de las personas "negras" y la "subjetividad negra" que se construye en las relaciones raciales. Existe, además, un interés por conocer esas dinámicas en las clases medias, sector socioeconómico que ha sido poco estudiado y que está en relación con los propósitos de la presente investigación. El autor concluye que la construcción de un modelo para pensar el racismo colombiano, implica agregar a las cuestiones históricas y estructurales, elementos de la sociabilidad que permitan entender los dispositivos de reproducción de un racismo cotidiano, no institucionalizado ni explícito. Por otro lado, entender "cómo se juega la igualdad" en la vida cotidiana, implica entender reglas más generales sobre la sociabilidad, especialmente los mecanismos de jerarquización cotidiana (Gil, 2010). Esto quiere decir que existen normas de prestigio social y circulación, basadas en prejuicios raciales, como el derecho de admisión a determinados lugares, la presentación personal, las interacciones cotidianas y las reglas endogámicas sobre el matrimonio.

Estas interacciones raciales cotidianas generan unos gastos emocionales y subjetivos para las personas estigmatizadas. En ese sentido, las personas "negras" que están en los "sectores medios" se ven obligadas a deconstruir un estereotipo racializado en el que, además de las representaciones referidas a la sexualidad, están también las de clase, expresadas en la cláusula común de "negro igual a pobre". 
Por lo tanto, es necesario hacer un proceso de des-identificación con ese estereotipo o, incluso, de distanciamiento con las otras personas "negras" de clases bajas (Gil, 2010), algo que es bastante similar a lo que en la literatura sobre el tema (Goffman, 1970; Cunin, 2003) se entiende como "estrategias de evitación".

Por último, el autor propone que el racismo en Colombia "no tiene lugar político" (Gil, 2010, p.49). Esto quiere decir que, públicamente y discursivamente, se ha negado su existencia como fenómeno social, por lo cual es difícil efectuar su denuncia, ya sea desde la academia o desde los movimientos sociales. Sin embargo, pensamos que esta no aceptación social del racismo como fenómeno social y la reticencia a plantearlo como asunto político, no necesariamente es una "característica colombiana" sino, justamente, un mecanismo de manifestación del racismo que otros estudios, en otros contextos nacionales, también han identificado (Fassin, 2002; Essed, 1991, 2010; Bonilla-Silva, 2011). De igual forma, en los últimos años, tanto movimientos sociales como investigadores en ciencias sociales y organizaciones multilaterales, han convergido en la necesidad de poner en un lugar público el tema del racismo; esto es, problematizarlo políticamente y visibilizarlo a nivel de la opinión pública (Mosquera \& Barcelos, 2007), en donde un subcampo de profundización ha sido el estudio del racismo en la escuela.

\section{El estudio del racismo en la Escuela: un promisorio campo de estudios}

Presentamos a continuación una breve revisión de algunos estudios que se han enfocado en el análisis del racismo en el medio escolar. Entendemos que es un cambio que ha presen- tado un auge de trabajos, por lo menos desde hace una década, con un énfasis importante en el estudio de los textos escolares (Soler, 2008) y la movilización de ideas racistas y prejuicios sobre los grupos étnicos en el país, pero también desde la movilización de estas ideas por parte de docentes y estudiantes, así como de la experiencia vivida del racismo en las interacciones escolares.

Una pionera en estos estudios ha sido María Isabel Mena (2009), quien ha desarrollado importantes reflexiones en torno al papel de los textos escolares para la enseñanza de la historia en la reproducción de estereotipos raciales, en el marco de la construcción identitaria nacional. Su estudio se enfoca en el análisis de la forma en que aparece ilustrada la historia de mujeres y hombres africanos y afrocolombianos en los textos escolares que los docentes utilizan para enseñar la historia de Colombia.

De sus resultados, se resaltan algunas características de la forma como se presenta a las comunidades afrocolombianas en las imágenes e ilustraciones incluidas en los textos de Historia, para los grados de tercero, cuarto y quinto de primaria. En primer lugar, la condición de esclavización parece caracterizar principalmente a estas poblaciones, por medio del uso ilustrado de grilletes, cadenas y el trabajo pesado (Mena, 2009). El segundo elemento -en la representación iconográfica de estas poblaciones- tiene que ver con su fuerte asociación con "lo racial", es decir que estas poblaciones se tratan de manera racializada. Es interesante que estas temáticas se presentan bajo el rótulo del "mestizaje", sin cuestionar ese proceso, como tampoco el carácter "racial" de las distintas poblaciones que empiezan a hacer parte de tal mestizaje (Mena, 2009). 
Otra característica tiene que ver con los movimientos de visibilización/invisibilización de la población afrocolombiana de la historia de Colombia. Esto obedece también a los registros institucionales que se han hecho de estas poblaciones, desde la institucionalidad estatal, a partir de los censos de población. Allí tampoco está presente la contribución de África y los africanos en la construcción de la identidad, la sociología y la historia colombianas (Mena, 2009).

Siguiendo esta senda de investigación, el trabajo de Castillo (2011) profundiza en el hecho de que las investigaciones demuestran que el discurso racista sigue vigente en los textos escolares de Ciencias Sociales, a pesar de haber transcurrido ya un largo período desde la promulgación de la Ley 70 para comunidades negras (en 1993). En su artículo, la autora analiza un interesante proceso de evolución del estigma y de resistencia sociopolítica, caracterizado por el surgimiento de manuales y textos escolares que se proponen contrarrestar el funesto impacto racista de los textos escolares tradicionales, por medio de la producción de material propio y autónomo de autoras y autores afrocolombianos, dirigido a un amplio sector educativo. La autora concluye que estos textos contra-hegemónicos logran articular dinámicas de autoreconocimiento,

en las historias locales, en las memorias colectivas, en las estéticas adaptativas, en esos innumerables modos como las afrocolombianidades se han inventado y reinventado a lo largo de los siglos, manifiestas -por ejemplo- en la tradición oral, pero, sobre todo, en las propias biografías de sus hombres y mujeres (Castillo, 2011, p. 71).
Desde la lingüística y el análisis de contenido, el trabajo de Correa (2010) pretende estudiar los modos narrativos mediante los cuales los niños, las niñas y los adolescentes en dos escuelas de la ciudad de Cartagena, perciben, comprenden y asumen el racismo. Estas personas narran historias de experiencias racistas en las cuales se reproducen las ideologías, los discursos y el posicionamiento actitudinal en relación con el racismo que circulan en la sociedad. Afirman -abiertamente- en sus relatos, que un niño o niña es negra o "muy negro" y que lo rechazaban por su color de piel. Por otro lado, "las víctimas" no se defienden y asumen, por lo general, una actitud de resignación. El autor concluye que estos discursos, contradictorios en algunos casos, son el producto de la fijación e interiorización cognitiva que los niños, niñas y adolescentes han asumido como racismo. En esa medida, la visión que se tenga del racismo en la infancia será decisiva a la hora de describir, interpretar y explicar los modos en que se articulan y escenifican los sentidos y asociaciones que se hacen de la "raza", la comprensión del concepto y la representación social ligada a esta noción (Correa, 2010).

Desde una perspectiva histórica, Meneses (2012) nos recuerda que la génesis de la educación y la escuela en Colombia, debe ser comprendida como:

El surgimiento de un sistema o dispositivo hegemónico y racializado, hecho por élites, que construye sentidos, discursos y percepciones sobre las diferencias para expropiar al otro de sí mismo, des-historizar, alienar, esclavizar, subalternizar, sub-desarrollar, occidentalizar, colonizar y dominar, sustentar un status quo sobre aborígenes y afrodescendientes (Meneses, 2012, p. 285). 
Para el autor, el sistema educativo y la escuela colombiana aún conservan "la estructura hegemónica, racializante, colonizadora, instaurada en los siglos XIX y XX ante la emergencia de los Estados-Nación y en el contexto histórico de la colonización americana" (Meneses, 2012, p. 285).

El interesante estudio de Valoyes (2015) muestra cómo esta condición racista de la escuela se puede reflejar en estereotipos sobre la performatividad de los estudiantes "negros" en el área de matemáticas. Los resultados confirman la existencia de una visión, según la cual, existe una jerarquía racial de las habilidades matemáticas, en la cual los estudiantes negros se ubican en la parte inferior ya que no cuentan con las disposiciones para aprender exitosamente esta disciplina (Valoyes, 2015).

Las representaciones negativas de los estudiantes negros elaboradas por los maestros entrevistados en el estudio se traducen en diferencias en las prácticas de enseñanza:

\footnotetext{
Estas se centran en el aprendizaje de procedimientos de rutina, en la memorización y en la solución de tareas de baja demanda cognitiva. Esto en contraste con las prácticas utilizadas para enseñar álgebra a los estudiantes ricos y mestizos quienes fueron posicionados como hábiles y capaces para el aprendizaje de esta disciplina. Luego, la relación entre ideologías y prácticas de enseñanza aporta a la comprensión de las experiencias y los resultados de aprendizaje de los estudiantes negros en Colombia (Valoyes, 2015, p. 201).
}

Finalmente el trabajo de Puertas (2010), de corte etnográfico en una escuela pública de Bogotá, encuentra que algunos de los niños y niñas de 3 a 5 años utilizan el color de la piel como característica para establecer una clasificación de personas y usan las etiquetas apren- didas de los adultos para denominarlas. Por otra parte, los niños de 7 y 8 años se refieren a características físicas solo al describir a compañeros o compañeras afrodescendientes, mientras que no lo hacen con estudiantes no racializados. Además, se refieren principalmente al color de la piel y el pelo, dos rasgos que han sido relevantes en la construcción histórica de la diferencia racial, si bien no adquirieren en estas edades las mismas significaciones (Puertas, 2010). Para la autora, la relevancia del color de la piel en la categorización social se mantiene hasta la adultez y es en estudiantes de 13 o 14 años cuando comienza a estar asociada con otros aspectos no físicos, "conformando un pensamiento racial”. Así mismo, encuentra que la existencia de estereotipos en las y los docentes fue generalizada y se mostró muy naturalizada y resistente al cambio (Puertas, 2010). Algunos de estos elementos coinciden con los hallazgos de nuestra investigación que presentaremos a continuación.

\section{Racismo cotidiano y experiencia vivida: consideraciones conceptuales y de método}

Los resultados que presentamos en este artículo se basan en un trabajo empírico realizado en la ciudad de Bogotá entre los años 2006 y 2009 , en el marco de una investigación doctoral sobre racismo en universidades, con una perspectiva comparativa entre las ciudades de París (Francia) y Bogotá (Colombia) (Quintero, 2013). El corpus principal de información se fundamenta en una serie de entrevistas en profundidad, realizadas en el segundo semestre de 2008 con 23 jóvenes universitarios que se autoidentifican como "afrocolombianos" o "negros" que hacían sus estudios de pregrado 
en Bogotá en universdiades tanto privadas como públicas. Algunos de ellos son provenientes de otras regiones del país, como la costa Pacífica o el Caribe colombiano, y otros tantos nacieron o llegaron muy pequeños a Bogotá, por lo que la mayor parte de su escolarización la hicieron en esta metrópoli.

Si bien uno de los propósitos principales de la investigación -de la cual surge este escrito- tenía que ver con la experiencia vivida del racismo por parte de estos estudiantes en las universidades, en los relatos biográficos surgió un elemento central y es que, en la mayoría de los casos, sobre todo para aquellos que tuvieron su escolarización temprana en Bogotá y en otras ciudades multiculturales, había un alto componente de racialización y de experiencias de racismo en la infancia, siendo la escuela un lugar preponderante donde se registra este tipo de situaciones que ellos consideraban, años después, como racistas. En ese sentido, lo que queremos presentar aquí son estos hallazgos centrados en sus recuerdos y relatos sobre sus experiencias de la infancia. Los relatos de estas personas entrevistadas sobre las experiencias de racismo vividas en el contexto universitario ya han sido objeto de publicaciones previas (Quintero, 2014, 2013a); por tal motivo, en la presente publicación nos centraremos en los hallazgos referidos exclusivamente al contexto escolar, como una forma de aportar al promisorio campo de estudios sobre el racismo en la escuela, del cual acabamos de presentar algunos de los trabajos.

Antes de seguir con el análisis, es menester informar sobre la perspectiva conceptual adoptada en la investigación y en el análisis de los datos. Para tal fin, es central la noción de racismo cotidiano acuñada por Philomena Essed (1991), la cual se ha constituido en una importante herra- mienta analítica en los estudios sobre racismo en los últimos años. Para esta autora holandesa, de origen surinamés, el racismo cotidiano es un proceso en el cual, en primer lugar, las nociones raciales son socializadas e incorporadas a "significados", por medio de los cuales, las prácticas sociales se vuelven "definibles y manejables"; en segundo lugar, las prácticas con implicaciones racistas se vuelven en sí mismas "familiares y repetitivas", por lo cual, en tercer lugar, las relaciones raciales y étnicas se "materializan y refuerzan" en las situaciones de la vida cotidiana, justamente a través de estas prácticas rutinarias o familiares (Essed, 1991).

Esta definición merece algunos comentarios adicionales. En primera instancia, que los individuos están insertos en procesos de racismo cotidiano, no solo con relación a procesos de dominación puramente raciales, sino que estos también se interrelacionan con condiciones, entre otras, de clase, género y edad, las cuales definen el contenido y la estructura de su vida cotidiana y, por ende, de las situaciones sociales más significativas. En segundo lugar, la noción de cotidianidad aquí acuñada no se refiere solamente a situaciones sociales de interacción cara a cara, sino también a aquellas con actores sociales no presentes, por ejemplo, con medios de comunicación, organismos públicos del Estado, empresas privadas, etc. (Quintero, 2013). Esto es importante tenerlo en cuenta, en la medida en que los estudios colombianos sobre racismo en la escuela muestran, justamente, que hay elementos institucionales que movilizan las ideas racistas, como en el caso de los libros de texto y en la continuidad de los prejuicios y estereotipos en varios de los actores del sistema escolar como lo son los docentes, estudiantes y padres de familia (Valoyes, 2015; Puertas, 2010; Correa, 2010; Meneses, 2012; Castillo, 2011). 


\section{Devenir "negro" en la escuela o las primeras experiencias de racialización}

Como ya ha sido explorado en otros estudios, la escuela es uno de los primeros contextos sociales en los cuales los niños y niñas aprenden a identificar y usar categorías raciales como categorías "válidas" de relación con los demás, así como de contrucción de una identidad de sí mismos racializada, ya sea que se trate de niños pertenecientes a grupos racialmente dominantes o dominados (Van Ausdale \& Feagin, 2001; Puertas, 2010).

Esta perspectiva supera las visiones tradicionales, de corte psicológista, sobre los niños y las niñas como recipientes vacíos que son llenados de contenido por medio de la socialización y la escolarización, quitándoles cualquier capacidad de agencia como sujetos de derechos ${ }^{3}$. Es así como en estos estudios se ha podido identificar que, al observar las interacciones de los niños y las niñas en sus grupos de pares, en efecto hay un desarrollo parcial en la comprensión de las dinámicas adultas frente a lo étnico-racial, pero esto no impide que los propios niños y niñas desarrollen sus propias dinámicas complejas en torno a lo étnico y lo racial (Van Ausdale \& Feagin, 2001, p. 16).

Es decir, los niños y las niñas, como sujetos activos que son, movilizan categorías raciales para comprender el mundo y comenzar a identificar un "nosotros" y un "ellos". Por ejemplo, Miguel, historiador de 37 años y estudiante de maestría en una universidad privada de élite en Bogotá, recuerda cómo conoció el racismo en una escuela en Valledupar, su ciudad natal ubicada en la región Caribe de Colombia:

Creo que me di cuenta que era negro en Valledupar ¿no? porque te lo hacen sentir en el colegio, porque en el salón no son muchos los que tienen el color de tu piel y, por supuesto, todo el racismo que hay detrás de eso, con los chistes, las bromas, etcétera y todo eso eh... y era una cosa que me molestaba mucho, es decir pues no tenía las herramientas para... articular un discurso alrededor de eso... yo era un niño de primaria y luego de secundaria; entonces era muy difícil... no tenía las herramientas para defenderme de ciertas cosas.

La racialización, en ese sentido, no es mayor ni menor, según el origen social de los estudiantes. Así pues, para Eduardo, 21 años, estudiante de economía en una universidad privada de élite, quien posee la mejor posición social de todas las personas entrevistadas, el cambio residencial de Buenaventura a Cali cuando entraba a tercero de primaria fue duro para él, particularmente por la discriminación y el racismo, los cuales vivió, particularmente, en la escuela:

Eso al comienzo fue muy duro, porque en Buenaventura, pues, la mayoría son afros, entonces uno se siente normal; pero uno ni siquiera sabía qué era la discriminación antes de irme a Cali, pero ya cuando fui a Cali, entramos a un colegio privado y éramos minoría étnica; entonces ya, cuando le colocaban a uno eh... apodos y todo eso, ya uno siente que uno es diferente y uno se choca, es un poco fuerte al comienzo.

Esta racialización, de la cual fue objeto Eduardo, le generó prevenciones que afectaron su socialización primaria, razón por la cual evitaba salir a jugar en su barrio y se mantenía en su casa, como una forma de "prevenir ese tipo de inconvenientes que sucedían en todo lado". Sus hermanos también vivieron esas situaciones, pero reaccionaban de otra forma, por lo cual tuvieron más inconvenientes y conflictos directos con otros estudiantes en el colegio. Según él, la formación académica que ha recibido le ha permitido cambiar 
de actitud y no aceptar pasivamente cualquier tipo de comentario o de insulto racista en su contra, sin que esto signifique entrar en confrontaciones violentas.

Por otro lado, Johanna, 20 años, estudiante de trabajo social en una universidad privada, recuerda que sus primeras experiencias de racismo ocurrieron igualmente en la escuela, manifestado principalmente mediante chistes y comentarios por parte de sus compañeros, en donde se reproducen estereotipos sobre la supuesta "flojera" de las personas "negras", "el mal olor" o "el bullicio". Todos estos estereotipos se van "mermando", según ella, cuando los otros se dan cuenta que es una de las mejores alumnas y que "es muy inteligente y es muy capaz de hacer los trabajos".

Así mismo, para Yadira, 18 años, nacida en Bogotá y estudiante de derecho en una universidad privada, su primera confrontación con el racismo ocurrió desde su infancia, particularmente en la escuela y en el colegio:

\footnotetext{
Primero empecé en transición, como a los tres años; entonces ahí era bien y la niña normal, la verdad, nunca sentí racismo ¿sabes?, hasta hace poco me di cuenta de que sí hay racismo... en el colegio... Espérate te cuento, en el colegio, pues, siempre era yo "la negrita", no me decían Yadira... sino "la negrita", "la morenita"; por ejemplo, iba a la casa de mis amigas, " $\mathrm{A}$ y hola la morenita es más linda!", pero no me decían Yadira ¿sí?... Entonces, como que una empieza a aceptar cosas y como que hay un momento en que, bueno, "me hace el favor, me respeta", pero ese respeto, realmente no está así, sino que una se cansa de escuchar siempre "la morenita" y una bueno, "¿mi mamá no me puso un nombre, mi papá no me puso un nombre?" eh... es como eso.
}

Pensamos que esta breve recopilación de relatos es ilustrativa de la importancia que ha tenido la escuela en la construcción social de las personas entrevistadas como "negras". Se trata -entonces- de una racialización inicial, en donde se hace una primera asignación identitaria como "negros" por parte del otro y en donde los estudiantes comienzan a negociar, aceptar o rechazar dicha asignación arbitraria. Muchas de las veces, como se aprecia en el anterior relato, los estudiantes no toman conciencia de este proceso inicial de racialización sino algún tiempo después de que éste comienza. Así mismo, si en un principio aceptaban esta asignación racial de forma pasiva, con el paso del tiempo expresan no aceptar situaciones flagrantes de racismo denotando reacciones más activas.

\section{Aprendiendo la "R": Racismo prosaico en las escuelas bogotanas}

En este artículo, queremos desarrollar la idea de la existencia de un "racismo prosaico" en las escuelas bogotanas. Como lo vimos en el acápite anterior, éste se constituye a partir de una temprana (en el sentido del ciclo de vida de los jóvenes afrocolombianos entrevistados) racialización violenta, simbólica y físicamente, que tuvieron que enfrentar los estudiantes entrevistados, con consecuencias que -tambiénson del orden psico-sociológico. Dicho de una manera más simple, es el momento en donde se dan cuenta que son definidos como "negros" por los otros, que esta asignación viene cargada principalmente de aspectos "negativos" que, en consecuencia, son tratados de manera diferente y que tienen que aprender a hacer frente a dicha asignación y tratos injustos, de los cuales, muchas de las veces, pueden surgir traumas de tipo psicológico que redundan en un deseo doloroso de negación del propio ser. 
Si bien la literatura especializada ha hablado más en términos de "racismo primario", creemos que esta noción no abarca la complejidad de la noción de racismo prosaico que queremos acuñar. Uno de los teóricos más influyentes sobre el racismo, ha propuesto una análisis del racismo en tres niveles, que harían parte de todo un proceso. En primer lugar, estaría el racismo primario, entendido como una reacción universal y casi natural ante cualquier cosa desconocida o persona extraña. Invariablemente, la primera reacción ante la extrañeza es la antipatía. Es entonces algo que se da de manera espontánea, por lo que no necesita ningún elemento o teoría que la inspire o la fomente (Taguieff, 1987, p. 64).

El racismo subiría un nivel de complejidad o sofisticación en lo que el mismo autor denomina como racismo secundario o racionalizado. Esto ocurre cuando una teoría es ofrecida e internacionalizada, la cual provee fundamentos lógicos para el resentimiento. El "otro" que se repela es entonces interpretado como "maligno" o intrínsecamente dañino para el grupo dominante (Taguieff, 1987, pp. 79). Finalmente, el racismo terciario o mistificador tiene en cuenta la presencia de los dos niveles precedentes pero se caracteriza por el desarrollo de argumentos de tipo cuasi-biológicos (Taguieff, 1987, pp. 90).

La noción de racismo prosaico que queremos proponer en este artículo tendría una cercanía analítica con la noción de racismo primario propuesta por Taguieff en la medida en que se puede pensar que los niños y niñas no racionalizarían muchos de los rechazos o estigmatizaciones que ejercen sobre otros infantes a su vez categorizados como "negros" y "negras". Sin embargo, una tal postura mantendría una visión limitada de la niñez, sustrayéndolos de su capacidad de agencia y de movilización de categorías sociales que utilizan no solo los adultos sino también los niños, dándoles su propio significado y sentido.

El carácter prosaico del racismo analizado obedece entonces a tres elementos importantes. El primero tiene que ver con los referentes de la racialización, o mejor, a los fundamentos que se utilizan para racionalizar la diferencia jerarquizada entre unos y otros: en este caso tiene que ver con una referencia muy simple y básica a las características físicas de los niños, o lo que Guillaumin (2008) denomina las características somáticas de las personas. Aquí no median necesariamente otras construcciones más "culturalistas" del racismo (Essed, 1991), como sí se puede presentar en otros contextos sociales y otros ciclos de vida. El segundo elemento tiene que ver con el carácter iniciático en la manifestación de este racismo, es decir en el momento específico las trayectorias de vida de los estudiantes, a saber: la socialización primaria en donde aprenden muchos de los códigos sociales y culturales para vivir en sociedad, que los lleva a adquirir una visión del mundo en donde hay "blancos" y "negros, entre otras categorías performáticas de la sociedad (por ejemplo las de "mujeres" y "hombres" aquí también son muy importantes). En tercer lugar, este racismo prosaico se caracteriza en sus formas de manifestación, por lo general sin mayores filtros sociales o de etiqueta, rayando con una suerte de ingenuidad, se presenta brutal, violento, vulgar, sin eufemismos.

En efecto, nuestra noción de racismo prosaico en la escuela surge del contraste de los hallazgos de nuestra investigación en el contexto universitario (Quintero, 2014, 2013a, 2013). En este último, el racismo pasa a ser algo que, 
desde el punto de vista discursivo, no es aceptado, por lo que adquiere formas de manifestación más sutiles, eufemizadas en cierto sentido. Esta característica contrasta con lo encontrado en el contexto escolar, en donde las referencias a "lo negro", como algo negativo y reprochable, no solo son explicitas sino que es explícita la asociación con la racionalización biologizante jerarquizante de la idea de la "raza", haciendo referencia por lo general a los rasgos físicos de los estudiantes: el pelo, el color de la piel, la asociación con los animales, la asignación constante de apodos, etc.

Con esta distinción no se quiere decir que uno u otro tipo de racismo sea "mejor" o "peor"; sencillamente se quiere denotar una diferencia cualitativa en su manifestación y características. Ambos son poderosos en su función de mantener relaciones sociales de dominación y de privilegios, así como de re-producción de desigualdades sociales; ambos son nefastos y dolorosos para las personas racializadas desde un punto de vista individual pero también colectivo.

La expresión violenta del racismo en el contexto escolar parece ser una constante en las experiencias vividas por los estudiantes entrevistados. El racismo prosaico se expresa mediante mecanismos diversos como lo son la Censura, la Desmotivación y la Intimidación. Esta última parece la más obvia, al relacionarse con la agresión física. No obstante, se constituye en la forma más explícita de toda una serie de formas precedentes que tienen que ver con comentarios, chistes o bromas de las cuales son objeto, sistemáticamente, los niños "negros" y que participan en el desarrollo de los mismos mecanismos.
La intimidación sirve, en algunas situaciones, para "poner las cosas en su sitio"; es decir, como un mecanismo para mantener el status quo de las relaciones jerárquicas entre estudiantes/docentes racialmente dominantes y estudiantes racializados; sirve como una advertencia a una posible subversión del orden, pero también como castigo o corrección frente a los intentos de resistir a dicha dominación, ya sea mediante estrategias de devolución del estigma o de denuncias ante las autoridades escolares sobre la situación.

En casi todas las situaciones específicas de agresión física del cual fue víctima un o una estudiante, también se identificó un mecanismo de Negligencia en el manejo o aceptación de una situación como discriminatoria o racista, constituyéndose en la contraparte simbólica (e institucional) de la violencia física. Esto obedece a que la posición subordinada de estos niños y de sus familias facilita mecanismos complementarios de Problematización por los cuales se tiende a culpabilizar a la víctima y, por consiguiente, a no tomar en serio las quejas o denuncias sobre los maltratos que están afrontando dichos estudiantes.

En cierta forma, el problema se evidencia una vez que la situación se convierte en algo violento, en donde, por lo general, también los mismos estudiantes racializados ejercen la violencia como una forma de protección o reacción frente al acumulado de maltratos que han venido recibiendo por parte de sus compañeros. En algunas ocasiones, simplemente no pasa nada; es decir, quienes ejercen el racismo no reciben una intervención pedagógica al respecto o no sufren consecuencias punitivas de ninguna índole. En otras ocasiones, sí son sancionados de alguna forma disciplinaria, pero también lo es el estu- 
diante racializado. Al final del proceso, queda una sensación, sin embargo, de culpabilización de la víctima, ya sea por no poner en consideración ni tomar seriamente sus quejas, como por las sanciones que le son impuestas cuando el estudiante -previamente ofendido- se defiende de forma violenta.

Desde un punto de vista sociológico, esto tiene consecuencias que se relacionan con otros mecanismos complementarios, como por ejemplo, el aislamiento, en la medida en que los estudiantes racializados sienten que son rechazados por los otros. Este aislamiento es característico de la banalización social que se puede hacer del racismo en la sociedad colombiana, en donde los chistes y las bromas son algo "no muy grave" y en donde, muchas veces, quien se queje del racismo por estas formas de expresión es, a su turno, acusado de racista y de intolerante, otro mecanismo frecuente del racismo cotidiano identificado en este estudio y el cual también está relacionado con la censura, como un mecanismo de acallar o reprimir una mirada o una voz crítica frente al estado de las cosas.

Valeria, 22 años, nacida en Pereira pero residente en el área metropolitana de Bogotá desde los 4 años de edad, estudiante de ciencias sociales en una universidad pública, recuerda que que fue sancionada en su colegio por agredir a unos compañeros, quienes llevaban mucho tiempo haciéndole matoneo, explícitamente racista. Al ver la gravedad de la situación y al presentar la queja ante el colegio por parte de su madre, quien consideró la sanción como injusta, generó otras acciones que, aunque bien intencionadas, también encarnan otros mecanismos del racismo en la escuela, como el paternalismo y la condescendencia institucionales:
Mi mamá fue y habló con el coordinador, con la rectora porque mi mamá decía - "Yo no entiendo por qué la tienen que expulsar, si ella actuó en defensa propia, el irrespeto no fue por parte de ella, porque si a ustedes los están agrediendo tres personas, pues ella no se va a dejar que la maltraten, ¿no?" - ¿Y qué decía la rectora? - Es que yo me acuerdo que en esa época estaba la transición entre el rector y una nueva rectora, entonces como el rector decía: - "No... yo la comprendo también; perdón por el descuido de no atender lo que su hija ya nos había dicho". Porque ya había pasado ante tales profesores, o sea, como que había quemado el conducto regular y no había podido hacer nada como más específico. Entonces, me acuerdo que, después eso, fue una charla de psicología, [por]que lo llevan a uno al psicólogo: - “¿cómo estás, cómo te sientes?”... O sea, lo que nunca hicieron antes, como de manejo [de] los derechos humanos, porque no existían; entonces, ya, después, era como la sobre-atención, ¿sí?, entonces mi mamá decía después, como al final de año, en la reunión de padres de familia, mi mamá decía que, después de los acontecimientos, que todo el mundo ya sabe fue como una sobre-atención, pero no se ve como un manejo o como una política institucional hacia el manejo de los derechos humanos, hacia el respeto por el otro ¿sí?, sino que el problema entonces se centró [solo en eso] y ya, no existía una política institucional dónde se tuviese en cuenta eso.

La sobre-atención a la cual hace referencia la estudiante y su madre tiene que ver con una sobrecompensación por parte de la institución hacia la estudiante, la falta de políticas educativas y programas escolares que prevengan y enfrenten el racismo; una realidad que no se puede tratar con paños de agua tibia; también puede obedecer a una reacción institucional para prevenir posibles demandas legales que, en efecto, había podido entablar la familia de la estudiante.

El paternalismo y la condescendencia se convierten, entonces, en un mecanismo del racismo cotidiano, en la medida en que refuerzan la posición subordinada de estudiantes "negros"; poco interesa si las acciones de alguien, o de 
una institución, en su conjunto, están motivadas por "buenas intenciones", pues éstas se fundamentan, igualmente, en la idea de una inferioridad latente de dichos estudiantes, cuyo corolario es la superioridad, también implícita, de quien ejerce esas buenas acciones.

En estrecha relación con este mecanismo está el de la inferiorización, el cual se expresa, de forma muy frecuente en el contexto escolar, mediante comentarios verbales, chistes y bromas e insultos. Consiste, como su nombre lo indica, en imponer una visión del mundo según la cual los estudiantes "negros" son inferiores a todos los demás, mediante una serie de elementos que son considerados importantes en el proceso de distinción entre personas y grupos sociales.

Allí parece que el prejuicio más común es el relacionado con las capacidades intelectuales de los estudiantes racializados, pues es en éste en donde se supone que estas capacidades se desarrollan, se (de)muestran y se plasman, por medio de las "buenas notas" y del "buen comportamiento". Esto último remite a que este mecanismo también se relaciona con unos supuestos estándares de comportamiento, léase valores morales, que "por su naturaleza", los estudiantes "negros" se supone no pueden tener.

¿Y por qué dices que esta profesora era racista?

Porque varias veces en el salón era... - "jay negrita, venga y ayúdeme aquí, ay negrita venga y usted..." por ejemplo cuando me regañaba, no me decía - "Yadira, ¿usted por qué hace tanta bulla o por qué habla tanto?..." sino - "¡Ay es que usted es más bullosa!... ¡es que negra tenía que ser!”, una vez me dijo.

De igual forma, la inferiorización se puede combinar y reforzar con otros mecanismos; es decir, para "hacerle ver al estudiante negro que se le considera inferior", se puede recurrir (como en el relato anterior) a una denigración cultural o también se puede deshumanizar o comparar con animales. Este último mecanismo puede manifestarse a través de un gran repertorio de formas de expresión en el contexto escolar. Puede ir de chistes y bromas, pasando por apodos, comentarios e insultos, hasta llegar a comportamientos de señalar o comentar las características físicas de los estudiantes "negros" (color de piel, pelo, nariz, etc.), mostrar una actitud de desprecio, displicencia o agresividad, o evitar el contacto físico o visual. En ese sentido, cuando se expresa por medio de apodos puede consistir en llamar cotidianamente al estudiante como "negro", en vez de llamarlo por su nombre de pila, o también en ponerle apodos que hagan referencia explícita a sus características físicas. Esta práctica tiene la consecuencia de deshumanizar al estudiante racializado, en la medida en que lo cosifica de forma estereotipada. Este mecanismo tiene unos efectos bastante dolorosos en los niños que lo experimentan:

¡Uy no!, pues al principio fue difícil porque, desde pequeña, me generaron un trauma tenaz, porque me decían dizque chorro de humo... una mano de apodos, así. Chorro de humo por el cabello. ¡Uy, fue tenaz! o sea todo eso fue tenaz, desde pequeña aún viviendo acá [en Bogotá], criándome con personas blancas, fue tenaz, fue algo horrible, horrible, porque los papás... y si no los papás eran los chinitos a uno, de la misma edad de uno, diciéndole cosas a uno, por el color, uy no; pero, entonces hubo un momento en el que sí fui muy agresiva y que yo dije - "no me la voy a dejar montar, iqué tal!" y mi papá era agresivo, entonces mi papá decía, - "la persona que le diga negra, me avisa y yo le doy en la mula". (Negrilla añadida).

El hecho de deshumanizar, mediante la atribución de apodos, está muy relacionado con la comparación de los estudiantes con la naturaleza 
de algunos animales. Es, por esta razón, que se ha decidido interpretarlo como un solo mecanismo, el cual se refleja en el nombre del mismo. Éste también es -quizás- el más ejemplarizante de este tipo de racismo prosaico, tan presente en ciertas escuelas bogotanas, pues son las características físicas las que primero llaman la atención en la racialización ejercida por niños y niñas mestizos, al no tener más referentes que los de su entorno infantil, facilitado en gran parte por los medios de comunicación, recurren a ellos para denominar esa realidad que les parece distinta, pero también inferior, y, ¿por qué no?, intimidante.

En ese sentido, el racismo se convierte en una forma básica de relacionarse con ese otro, la cual sirve para poner en claro cuáles deben ser las relaciones de jerarquía entre los infantes, constituyendo así otro elemento de su carácter prosaico. Los apodos se refuerzan mutuamente con los chistes y las bromas y con el señalamiento o comentario sobre sus características físicas, en donde también se deshumaniza e inferioriza a los estudiantes "negros", mediante símiles con animales, con objetos o personajes de la televisión. Todo esto, también, con efectos traumáticos en los niños que reciben las burlas, como se puede apreciar en el siguiente relato de Humberto, 26 años, estudiante de ciencias sociales en una universidad pública, oriundo de Bogotá:

Mi mamá es una persona muy dedicada... muy dedicada con nosotros, digamos que, en algún sentido, de sobreprotección de pequeños, porque, digamos que el contexto de la ciudad es un contexto totalmente diferente, a ella le afectaba mucho el racismo que se aplicaba con nosotros en ese tiempo de niños; en ese sentido, nosotros no salíamos mucho, a mi mamá no le gustaba que estuviéramos como en parques, porque vivía preocupada por eso. Trataba de todo darnos... de siempre estar como dentro del núcleo familiar. Un ejemplo: un 31 de octubre, ella prefería comprar los dulces, comprar cajas, paquetes de dulces y dejarnos en la casa; casi no le gustaba que...una vez yo no me acuerdo, sí... yo creo que a mí o a mi hermano lo disfrazaron de Superman o de Batman, es que no... yo no recuerdo cuál de los dos era, porque no... entonces hubo ahí risas, burlas, por la cuestión étnica; entonces, desde ahí mi mamá...

¿Y quién se burlaba?...

La gente, los niños del colegio... pues como: - "jun Superman negro!", un Batman, ¿sí me entiendes? Entonces, desde ahí no le gustaba mucho el contexto de la sociedad bogotana..." (Negrilla añadida).

En el anterior relato está presente un mecanismo que merece especial atención, pues cumple una función importante en la reproducción del racismo en las sociedades contemporáneas. Se trata de la reactualización del racismo con base en referentes sociales o culturales. Éste consiste en la persistente actualización del racismo, entendido como ideología, como relación social de poder y como práctica cotidiana, con base en referentes sociales, históricos y culturales, tomados de situaciones o temas de actualidad. Por ejemplo, la producción constante de chistes y bromas con referentes culturales contemporáneos, que reproducen las ideas racistas sobre una supuesta inferioridad moral o intelectual de las personas "negras".

Lo vemos con las referencias a personajes de programas infantiles de televisión o de cómics, como Dragon Ball Z y Superman. Este último se constituyó en el fundamento de la burla y las risas, pues el arquetipo dicta que este personaje, como símbolo del poderío masculino y occidental, es "blanco" y no puede ser "negro"; lo cual también está relacionado con el mecanismo de Blancocentrismo-etnocentrismo, en donde lo blanco y lo europeo son vistos como el centro del mundo y el punto de vista moral y ético de comportamiento. 


\section{Discusión final: caracterizar el racismo para poder combatirlo}

Qusiéramos terminar este artículo con una idea enfática: no hay racismo soft o racismo bien intencionado. El racismo, entendido como un tipo específico de relación social de dominación, termina legitimando estructuras de desigualdad social a partir de una idea vaga y difusa de "raza". Esta idea es la justificación de la desigualdad y no la causa. Sin embargo, el racismo, como todo sistema de relaciones sociales, no es unívoco y se configura de manera particular en los contextos sociales y en los distintos campos sociales. Es lo que hemos propuesto hacer con este artículo en relación con la manifestación del racismo en contexto escolar, a través de la reflexividad desarrollada por jóvenes afrocolombianos y afrocolombianas que recordaron y comentaron sus experiencias del pasado como escolares, resignificándolas desde su presente como estudiantes universitarios.

En el campo de estudios sobre el racismo, es común tratar de buscarle apellidos a este fenómeno: racismo cultural, racismo biológico, neo-racismo, entre tantas otras acepciones por cada investigador o investigadora que se enfrentan a este objeto. En nuestro caso, aunque no hubiéramos podido escapar a esta tentación bautizadora, o mejor, esta necesidad para poder entender mejor el fenómeno, la categoría surgió de los datos mismos; en especial del contraste entre lo encontrado en la escuela $y$ en la universidad por parte de las mismas personas entrevistadas.

La noción de racismo prosaico no significa una manifestación más "benéfica" o "banal" del racismo por el hecho de ser agenciada por niños y niñas. Su característica principal es quizás el hecho de ser estructurante, y a la vez estructurado, de/por las relaciones sociales racializadas y racializantes ${ }^{4}$, reproduciendo la noción de la diferencia social a través de la idea de "raza". Lo que significa ver, pensar, sentir y actuar orientados por la diferencia entre un ellos "negros/negras" y un nosotros "blancos/blancas"; asociando tanto una valoración, como un estatus y una dignidad distintos, los segundos ubicándose por encima de los primeros.

Lo anterior nos remite a otro elemento importante en la manifestación de este racismo prosaico: su carácter profundamente violento desde el punto de vista simbólico (Bourdieu, 1998). Este ha significado que en la escuela se presenta una temprana racialización para algunos de las y los estudiantes "negros" entrevistados. De igual forma, el carácter abierto y muchas veces descarnado de estas expresiones racistas no deja de estar exento de consecuencias emocionales en los estudiantes. No en vano salieron a relucir en las entrevistas, a pesar de que fueron situaciones vividas hace muchos años atrás. El racismo en la escuela está, entonces, en la base de la constitución de una diferencia radical, aquella de las personas racializadas, configurándose en un tipo de relación social de poder difícil de cuestionar, sobre todo por aquellos que tienen la posición dominante en la sociedad, es decir aquellos que no son marcados racialmente.

Como se ha demostrado en otros estudios recientes (Mena, 2010), el racismo está bien presente en el sistema escolar bogotano. A pesar de las constantes voces en contra de esta idea, expresadas principalmente por ciertos sectores políticos, en los discursos de instituciones públicas o personalidades políticas, e incluso en 
los propios docentes y personal directivo de los colegios (Mena, 2010), el presente trabajo se propuso aportar a la comprobación de una realidad social existente, así como la necesidad de seguir trabajando desde las ciencias sociales para comprender mejor el fenómeno y vislumbrar posibles cambios de trasformación social.

No obstante, aunque el racismo y las discriminaciones fundamentadas en la idea de "raza" son una realidad social en nuestro sistema educativo, en especial en el ámbito escolar, como espacio-tiempo significativo para las niñas, los niños y jóvenes, pensamos que éste no es el asunto central que surge de nuestra investigación, pues nuestro propósito no era el de "demostrar" la existencia del racismo en la escuela. Reducir nuestra investigación a la misma habría significado caer en la lógica del juicio (Wacquant, 1997), con el propósito de emitir conceptos valorativos de las sociedades analizadas, estilo "la sociedad bogotana es racista". Por el contrario, lo que queremos entender es cómo funciona el racismo como un sistema de relaciones sociales de poder, que se legitima a sí mismo con la excusa de la idea de "raza" y que tiene consecuencias concretas no solo en las relaciones escolares mismas, sino en las futuras trayectorias educativas y sociales de niñas, niños y jóvenes racializados.

Lo identificado en relación con el racismo en la escuela nos demuestra cómo los procesos de racialización se originan muy temprano en la primera infancia, acorde con algunos estudios que han mostrado -específicamente- que las categorías raciales hacen parte de los marcos cotidianos de interacción de los niños y niñas, coadyuvando a la creación de una identidad entre un "yo/nosotros" y un "él/ellos" racializados (Van Ausdale \& Feagin, 2001).
Esto es cierto no solo para el temprano proceso de hetero-asignación categorial como "negros", sino también para el carácter repetitivo de las situaciones cotidianas, así como el carácter acumulativo de los procesos de discriminación, a lo largo de sus trayectorias vitales y en su experiencia como estudiantes universitarios, período de sus vidas en los que fueron entrevistados los estudiantes co-partícipes de esta investigación.

Recordemos que, con base en la ideología de la meritocracia, el sistema educativo se legitima a sí mismo, legitima los procesos de selección de los estudiantes y los tratamientos diferenciales aplicados a algunos de ellos a partir de prácticas institucionalizadas, como el ejercicio de la autoridad escolar, los exámenes, las evaluaciones, los concursos, etc. La meritocracia es el fundamento para escoger no solo quiénes ingresan, sino también quiénes salen (se gradúan) y a qué sectores de la economía y el trabajo van a parar, con niveles salariales disímiles de acuerdo con la "calidad" del egresado. Se trata, entonces, de una elección de "los mejores entre los mejores" que se repite a medida que aumenta el nivel educativo en una suerte de profecía autocumplida.

La dominación socio-racial en la educación se fundamenta, en ese sentido, en la arbitrariedad del valor meritocrático que, en teoría, no daría lugar a lo arbitrario. Ésta es doble y siempre tiene por consecuencia la minorización de los estudiantes "negros" en el sistema educativo. El mérito en la educación obedece, entonces, al orden de lo intelectual y del manejo de los conocimientos establecidos como válidos y legítimos. Estas "cualidades" constituyen así el telón de fondo del proceso de minorización de los estudiantes "negros" 
que comienza en la escuela en donde la idea de la "raza negra" viene acompañada entonces de una supuesta inferioridad intelectual y moral de los estudiantes "negros".

El desafío educativo en Colombia es poder desmontar estos procesos de re-producción de las ideas racistas, por lo menos generar ambientes escolares libres de racismo o en donde cualquier manifestación de este tipo sea sistemáticamente cuestionada, explicada, recontextuaizada y resignificada. De lo contrario parece que estaremos inexorablemente condenados y condenadas a repetir la misma historia, con otros personajes y en otros tiempos.

\section{Notas}

\footnotetext{
${ }^{1}$ En este artículo se utilizarán las categorías de asignación étnico racial como "negro/a", "afrocolombiano/a", "raizal del Archipiélago", "blanco/a", etc. entre comillas para recordar el carácter socialmente construido, político e ideológico de la idea de "raza".

${ }^{2}$ Un verdadero campo de estudios con una gran cantidad de trabajos académicos producidos y cuyo trabajo de sistematización, análisis y síntesis en profundidad estaría por fuera de los límites del presente escrito. Para tal fin, retomamos algunos de los principales
}

\section{Referencias bibliográficas}

Agier, M. \& Hoffmann, O. (1999). Les terres des communautés noires dans le Pacifique colombien. Interprétations de la loi et stratégies d'acteurs. Problèmes d'Amérique Latine, (32), 17-42.

Arocha, J. et al. (2007). Elegguá y respeto por los afrocolombianos: una experiencia con docentes de Bogotá en torno a la Cátedra de Estudios Afrocolombianos. Revista de Estudios Sociales. Dossier Raza y Nación (II), (27), 94-105.

Bonilla-Silva, E. (2011). ¿Qué es el racismo? Hacia una interpretación estructural. En Mosquera Rosero-Labbé, C., LaóMontes, A. \& Rodríguez Garavito, C. (Eds.), Debates sobre ciudadanía y políticas raciales en las Américas Negras (pp. 649-698). Bogotá: Universidad Nacional de Colombia. Facultad de Ciencias Humanas. Centro de Estudios Sociales-CES/ Universidad del Valle.

Bourdieu, P. (1998). La Domination Masculine. Paris: Seuil. (1997). Razones Prácticas. Sobre la teoría de la acción.

Barcelona: Anagrama.

Castillo, E. (2011). "La letra con raza, entra". Racismo, textos escolares y escritura pedagógica afrocolombiana. Pedagogía y Saberes, (34), 61 - 73.

Correa, C. (2010). Estudio discursivo del racismo narrado por niños: "Erase una vez un niño llamado Jorge, él era muy negro y por eso nadie en su colegio quería jugar con él". (Trabajo de grado de lingüística). Universidad de Cartagena, Cartagena.

Cunin, E. (2003). Identidades a flor de piel. Lo "negro" entre apariencias y pertenencias: categorías raciales y mestizaje en y más acabados trabajos de "estado del arte" que ha realizado el antropólogo colombiano Eduardo Restrepo (2004, 1999).

${ }^{3}$ Para una importante y rigurosa revisión y crítica a estas perspectivas sobre la infancia, véase el ya citado libro de Van Ausdale \& Feagin (2001).

${ }^{4}$ Siguiendo un poco la perspectiva analítica que nos permite el concepto de habitus en la teoría de Bourdieu (1997).

Cartagena. Bogotá: ICANH-Universidad de los Andes-IFEAObservatorio del Caribe Colombiano.

De la Hoz, D. (2012). Estudio de la apreciación en relatos sobre discriminación percibida por afrodescendientes en Cartagena. En Fonseca, C. (Comp.) Racismo: miradas cruzadas (pp. 87-121). Cartagena de Indias: Editorial Universitaria.

Essed, P. (2010). Hacia una conceptualización del racismo como proceso. En Hoffmann, O. \& Quintero, O. (Coord.), Estudiar el racismo. Textos y herramientas. Documento de Trabajo № 8 / Document de Travail № 8 (pp. 129-169). México: Proyecto AFRODESC / EURESCL. (1991). Understanding everyday racism. An interdisciplinary theory. Newbury Park, California: Sage Publications.

Fassin, D. (2002). L'invention française de la discrimination. Revue Française de Science Politique, 52 (4), 403-423.

Fonseca, C. (2009). Aproximaciones teóricas y metodológicas a los estudios del racismo. Documento de Trabajo № 6 / Document de Travail № 6. Cartagena: Proyecto AFRODESC / EURESCL.

Fonseca, C. \& Badrán, F. (Eds.). (2012). Racismo: miradas cruzadas. Cartagena de Indias: Editorial Universitaria-Universidad Nacional de Colombia.

Gil, F. (2010). Vivir en un mundo de 'blancos'. Experiencias, reflexiones y representaciones de 'raza' $y$ clase de personas negras de sectores medios en Bogotá D.C. (Tesis inédita de maestría). Universidad Nacional de Colombia, Bogotá.

Goffman, E. (1970). Ritual de la interacción. Buenos Aires: 
Tiempo contemporáneo.

Guillaumin, C. (2008). Práctica del poder e idea de naturaleza. En Curiel, O. \& Falquet, J. (Comp.). El patriarcado al desnudo. Tres feministas materialistas: Colette Guillaumin -Paola Tabet - Nicole Claude Mathieu (pp. 19-56). Buenos Aires: Brecha lésbica.

Hoffmann, O. (2004). Communautés noires dans le Pacifique colombien. Innovations et dynamiques ethniques. Paris: Karthala- IRD.

Langebaek, C. \& Leal, C. (2007). Presentación. Revista de Estudios Sociales. Dossier Raza y Nación (II), (27), 11-13.

Meertens, D., Viveros, M. \& Arango, L. (2006). Discriminación étnico-racial, desplazamiento y género en los procesos identitarios de la población negra en sectores populares de Bogotá. Bogotá: Clacso-Crop, Cidse.

Mena, M. (2010). Si no hay racismo no hay Cátedra de Estudios Afrocolombianos. Bogotá: Proyecto dignificación de los y las afrodescendientes y de su cultura en Colombia-AECID-SED.

(2009). La ilustración de las personas afrocolombianas en los textos escolares para enseñar Historia. Revista Historia Caribe, (15), 105-122.

Meneses, Y. (2012). Maestro: ¿Promotor de la cultura y/o promotor del racismo? Reflexiones en torno al surgimiento de la escuela en el Estado-Nación, el racismo en la escuela y la condición maestro en el Estado pluriétnico y multicultural. Revista Plumilla Educativa, (9), 281-296.

Mosquera, C. (2007). Lecturas críticas de los talleres de salud sexual y reproductiva y de fortalecimiento cultural desarrollados con mujeres negras desterradas por el conflicto armado en Colombia. Revista de Estudios Sociales. Dossier Raza y Nación (II), (27), 106-121.

(1998). Estrategias de inserción de la población negra en Santafé de Bogotá. 'Acá en Bogotá no se veían negros'. Bogotá: Observatorio de Cultura Urbana-Instituto Distrital de Cultura y Turismo.

Mosquera, C. \& Barcelos, L. (Eds.). (2007). Afro-reparaciones: Memorias de la Esclavitud y Justicia Reparativa para negros, afrocolombianos y raizales. Bogotá: Universidad Nacional de Colombia.

Puertas, M. (2010). Del color de la piel al racismo. Prácticas y representaciones sobre las personas afrodescendientes en el contexto escolar bogotano. Un estudio de caso. (Tesis inédita de maestría en antropología). Universidad Nacional de Colombia, Bogotá.

Quintero, O. (2014). El racismo cotidiano en la universidad colombiana desde la experiencia vivida por los estudiantes negros en Bogotá. Revista Universitas Humanística, № 77, ene-jun, pp. 71-94. (2013a). Discriminaciones raciales cotidianas como forma de construcción de masculinidades en la universidad. Educar en la equidad. Boletina Anual № 2. Escuela de Estudios de Género, Universidad Nacional de Colombia, pp. 26-33.
(2013). Racisme et discrimination à l'université. Lectures croisées des sociétés Française et Colombienne à partir de l'expérience vécue des étudiants Noirs à Paris et Bogota. (Tesis inédita de doctorado en Sociología). Université de Haute Bretagne-Rennes 2, Rennes.

Restrepo, E. (2004). Hacia los estudios de las colombias negras. En Rojas, A. (Comp.). Estudios afrocolombianos. Aportes para un Estado del Arte (pp. 19-57). Popayán: Editorial Universidad del Cauca. (1999). Compilación bibliográfica sobre poblaciones negras en Colombia. Documentos de Trabajo (43). Cali: Cidse-Univalle.

Restrepo, E. \& Rojas, A. (2008). Afrodescendientes en Colombia: Compilación bibliográfica. Popayán: Universidad del Cauca.

Rodríguez, C. et al. (2008). El derecho a no ser discriminado. Primer informe sobre discriminación racial y derechos de la población afrocolombiana. Bogotá: Universidad de los Andes- Facultad de Derecho.

Soler, S. (2008). Pensar la relación análisis crítico del discurso y educación. El caso de la representación de indígenas y afrodescendientes en los manuales escolares de ciencias sociales en Colombia. Discurso \& Sociedad, 2(3), 642-678.

Taguieff, P-A. (1987). La force du préjugé. Essai sur le racisme et ses doubles. París: La Découverte/Gallimard.

Universidad de Texas (2007). Unfulfilled promises and persistent obstacles to the realization of the rights of Afro-Colombians. A report on the Development of Ley 70 of 1993. Austin: Rapoport Center for Human Rights and Justice.

Urrea, F. (2011). La conformación paulatina de clases medias negras en Cali y Bogotá a lo largo del siglo XX y la primera década del XXI. Revista de Estudios Sociales (39), abril, 24-41.

Valoyes, L. (2015). "Los negros no son buenos para las matemáticas”: Ideologías raciales y prácticas de enseñanza de las matemáticas en Colombia. Revista CS (16), 169-206.

Van Ausdale, D. \& Feagin, J. (2001). The first R. How children Learn Race and Racism. Maryland: Rowman \& Littlefield Publishers.

Viáfara, C. \& Urrea, F. (2006). Efectos de la raza y el género en el logro educativo y estatus socio-ocupacional para tres ciudades colombianas. Desarrollo y Sociedad, 58, 115-163.

Viveros, M. (2007). Discriminación racial, intervención social y subjetividad. Reflexiones a partir de un estudio de caso en Bogotá. Revista de Estudios Sociales. Dossier Raza y Nación (II), (27), 106-121.

Viveros, M. \& Urrea, F. (2007). "Raza”, género y ascenso social: La experiencia de las clases medias negras en Colombia (Un estudio de caso en Bogotá y Cali). Proyecto de Investigación. Bogotá: CesCidse-Colciencias.

Wacquant, L. (1997). For an analytic of racial domination. Political Power and Social Theory, 11, 221-234. 